\title{
Laser Spinning of bioactive glass nanofibers**
}

By Félix Quintero*, Juan Pou, Rafael Comesaña, Fernando Lusquiños, Antonio Riveiro, Adrian B. Mann, Robert G. Hill, Zoe Y. Wu, and Julian R. Jones

[*] F. Quintero, J. Pou, R. Comesaña, F. Lusquiños, A. Riveiro. Department of Applied Physics, ETSII, University of Vigo 36310 - Vigo (Spain)

E-mail: fquintero@uvigo.es, F. Quintero Corresponding-Author

\section{A. B. Mann}

Materials Science \& Engineering Dept, Rutgers University

Piscataway, NJ 08854 (USA)

R. G. Hill, Z. Y. Wu, J. R. Jones,

Department of Materials, Imperial College London, South Kensington Campus London SW7 2AZ (UK)

[**] Authors wish to thank Dr. Benito Rodríguez from CACTI (University of Vigo) for his help with the TEM characterization. This work has been partially supported by the Spanish Government, (CICYT MAT2006-10481 and FPU program AP2006-03500 grant), and by Xunta de Galicia (PGIDIT05TMT30302PR, PR405A2002/11-0, INCITE07PXI303112ES and INCITE08PXIB303225PR).

Keywords: bioactive glass, nanofibers, laser processing.

The production of nanofibers of bioactive glass by laser spinning is reported. The technique yields a great quantity of free-standing fibers in the form of a mesh of disordered intertwined fibers. The method does not rely on chemical processing and does not need any chemical additive. It involves melting of a precursor material with tailored composition, which makes it possible to produce nanofibers from materials with which conventional melt drawing techniques cannot be used. Herein, the production of 45S5 Bioglass ${ }^{\circledR}$ nanofibers is reported for the first time. The process is very fast (nanofibers of several centimeters are grown in a fraction of a second), without the necessity of post heat treatments, and no devitrification was observed as a result of 
the laser spinning process. The morphology, composition and structure of the nanofibers were characterized and their bioactivity assessment was carried out by immersion in SBF. This technique provides a method for the rapid production of dense glass nanofibers which can be employed as bioactive nanocomposite reinforcement, as a synthetic bone graft to replace missing bone, or to produce $3 \mathrm{D}$ structures for use as scaffolds for bone tissue engineering.

\section{Introduction}

A considerable variety of materials have been investigated to enhance bone regeneration where it has suffered any damage or disease. While bio-inert materials were preferred in the past for implantation, over the last decades their applications demand extensive research on bioactive materials capable of promoting cell differentiation, proliferation, three-dimensional growth and integration to surrounding bone. ${ }^{[1-3]}$ Selection of the proper geometrical architecture of scaffolds for tissue engineering is essential to achieve mechanical requirements, improve nutrients transport and biofactor delivery. ${ }^{[4]}$ Currently, porous foams and fibrous meshes are common motifs. ${ }^{[5]}$

Most of the work on fibrous meshes for tissue scaffolds deals with the generation of polymer fiber by electrospinning. Some researchers have also suggested the potential of bioactive glass fibers as scaffolds for tissue engineering and investigated their production and bioactivity with interesting results. Two main different techniques were initially employed to produce the glass fibers: spraying ${ }^{[6,7]}$ or dry-spinning ${ }^{[7,8]}$ of a solgel precursor or by directly melting the glass and forming it through a platinum bushing. ${ }^{[9-14]}$ Typical diameters of these fibers range from one micron up to several tens of microns. All of these works concluded that these kinds of scaffolds have the potential to direct and mediate cell growth as tissue engineering constructs in bone regeneration 
with different degrees of bioactivity depending on the composition, microstructure and surface features. The 45S5 bioactive glass is the most intensely studied and has been extensively distributed for clinical use (Novabone Products LLC, Alachua, Fl.). However, 45S5 glass cannot be easily drawn into fibers due to devitrification that occurs during the fiber drawing process, which will affect its bioactivity. For this reason, less fragile compositions with higher silica content were used, which can be fiberized and which can be tailored to provide controlled degradation but are still less bioactive than the $45 \mathrm{~S} \%$ glass. ${ }^{[9-13]}$ Alternatively, the techniques based on a sol-gel precursor offer a higher flexibility to vary composition but they produce fibers with lower strength ${ }^{[15]}$ and rely on the utilization of chemical solvents. On the other hand, the laser spinning technique has been demonstrated to produce fibers from a wide range of compositions, including non-fiberizing fragile melts, with no chemical additives involved in the process. ${ }^{[16,17]}$

Recent publications stress the importance of nano-scale architectures, surface patterns and stimuli to enhance the performance of scaffolds for tissue engineering. ${ }^{[18]}$ The extracellular matrix in living tissue provides a natural web of intricate nanofibers to support cells and strongly influences changes in cell shape that affect the differentiation process. Furthermore, nanoscale alterations in topography elicit diverse cell behavior. Therefore nanofibrous structures with controlled architecture have excellent potential to mimic natural extracellular matrix and to be used as scaffolds for tissue growth. The first approach took advantage of the well-known electrospinning technique for the production of nanofibers made of bioactive polymers. ${ }^{[19,20]}$ More recently, electrospinning of sol-gel precursor was applied for the production of nanofibrous meshes of bioactive glass demonstrating their potential as a novel biomaterial for tissue engineering. ${ }^{[21,22]}$ 
Herein we propose a new technique to produce nanofibers of bioactive glass in the form of intertwined disordered structures which may be used as scaffolds or as reinforcement in polymeric matrix composites: laser spinning. The results on the production of two different compositions of bioactive glass nanofibers by laser spinning are presented. Specifically, we report the first amorphous nanofibres produced from 45S5 bioactive glass. We also carried out bioactivity tests to analyze their solubility and reaction to simulated body fluid, showing the potential of this technique to produce bioactive glass nanofibrous meshes with tailored compositions to adjust their bioresorption and ion release.

\section{Results and discussion}

\subsection{Production and characterization of Bioglass ${ }^{\circledR}$ nanofibers}

Laser spinning involves melting a small volume of the precursor ceramic material at very high temperatures using a high power laser. Simultaneously, a gas jet of very high velocity from a supersonic nozzle is used to blow the molten material. The molten ceramic is initially a pendent drop, but under the action of the gas jet it is quickly stretched and cooled by the gas (Figure 1). Because of the speed of the cooling the final structure is amorphous, hence the bioceramics produced are essentially bioglasses. This process occurs at very high speeds and as a result the elongation of the molten material can produce fibers with extraordinary ratios of length to diameter $(1,000,000: 1)$ in a fraction of a second. The relative movement between the plate of precursor material and the laser beam produces a cut which continuously feeds molten material to the process. In this way, a dense mat of these glass nanofibers can be formed in a matter of minutes. The precursor materials employed to produce the glass fibers were a series of glass plates with the compositions of Bioglass ${ }^{\circledR} 45 \mathrm{~S} 5$ and 52S4.6 (Table 1). Several pieces 
of each glass composition were produced by means of the traditional method of glass melting. They were subsequently processed by means of Laser Spinning yielding a cotton-like mesh of micro- and nanofibers with good flexibility and consistency which allow their easy manipulation. Figure 2.a presents a field-emission scanning electron microscopy (FESEM) image showing an overall view of the typical appearance of the as-produced fibers. They form a disordered mesh of intertwined fibers with different diameters, typically in the range from tens of nanometers up to several microns. However, each fiber has a diameter that is almost perfectly uniform along its whole length with a distinct cylindrical shape (circular cross-section). An interesting property of these mats is that the fibers appear completely separated, there is not adhesion among different fibers. Consequently, they can be easily dispersed in solution and, eventually, it will be possible to use constrained flow techniques to align them and shape into highly ordered structures. Analyses performed by means of Electron Diffraction in Transmission Electron Microscope (TEM) demonstrate that the fibers are completely amorphous and this was independent of the microstructure and composition of the precursor material. Figure 2.b shows a TEM micrograph of a nanofiber with the composition 52S4.6 including the corresponding selected area electron diffraction (SAED) pattern. The nanofibers appear fully dense and solid, just as the fibers drawn from the melt obtained by conventional methods, which present high tensile strength thanks to their forming process.

The primary concern with regards to the composition of the fibers was to determine if they maintain exactly the same chemical composition as the precursor glass. Previous analyses and modeling of the physical process for fiber formation revealed that maximum temperatures in excess of $2000 \mathrm{~K}$ can be reached in the melt. ${ }^{[16]}$ For this reason we hypothesized that the most likely alteration in the composition might be 
depletion in the more volatile species such as alkali metals. Therefore, comparison between the composition of the precursor material and that of the fibers were undertaken using X-Ray Fluorescence (XRF). The same analysis conditions and sample preparation were used in each case to avoid any distortion from differences in sample quantity, size or morphology. Semi-quantitative analyses were performed, since no suitable appropriate standard of similar form for calibrating the XRF measurements were available. However, this method yields a reliable comparison of the relative proportions in the glasses and their corresponding fibers.

The results of the analyses in molar percentage are presented in Table 1. As a general conclusion for these results it can be pointed out that there is a slight depletion of the most volatile species, $\mathrm{Na}_{2} \mathrm{O}$ and $\mathrm{P}_{2} \mathrm{O}_{5}$, in the composition of all the fibers in comparison with the precursor glass plates. Concurrently, the less volatile species, $\mathrm{SiO}_{2}$ and $\mathrm{CaO}$, present a similar increase as a consequence. Notwithstanding, the differences in both pairs of compositions were very small and would have a minimal influence on the bioactivity of the glass. This good correlation between the composition of the plates and the fibers may be due to the high cooling rates, since there is no time enough for diverse vaporization of the species, a characteristic which is usual in high energy laser processes.

During the formation of the fibers by laser spinning, the molten glass undergoes rapid heating to high temperatures, followed by extremely high cooling rates and elongational stresses. These are potential reasons to think a change of the structure of the glass may occur. For example the fast cooling rates may quench in silicate and phosphate structures normally found in the melt and in Molecular Dynamic Simulations where the effective quench rates are extremely rapid. ${ }^{[23]}$ This could a wider distribution of $\mathrm{Q}$ species in the silicate structure which is closely related to its bioactivity. ${ }^{[24]}$ For this 
reason, and with the additional objective of reinforcing the compositional characterization, an analysis by magic angle spinning NMR (MAS-NMR) was carried out to compare both the precursor glass with the fibers obtained from them. Approximately $0.4 \mathrm{~g}$ of each sample were ground to the same size and ${ }^{29} \mathrm{Si}$ and ${ }^{31} \mathrm{P}$ spectra were taken on the four samples (two bulk and two fibers). The spectra for the composition of glass 45S5 from the bulk and from the fibers show a very similar broad asymmetric peak at around $79.5 \mathrm{ppm}\left({ }^{29} \mathrm{Si}\right)$ with full width at half maximum (FWHM) around $6.3 \mathrm{ppm}$ (Table 2). There was no broadening of the ${ }^{29} \mathrm{Si}$ peak that might indicate a wider distribution of $\mathrm{Q}$ species accompanying rapid quenching from the melt. Both samples show a very similar structure with ${ }^{29} \mathrm{Si}$ chemical shift characteristic for $\mathrm{Q}^{2}$ species (silica tetrahedra containing 2 bridging $\mathrm{Si}-\mathrm{O}-\mathrm{Si}$ bonds), as expected from their compositions. $^{[24,25]}$ Consequently, we assume the number of non-bridging oxygens / silica tetrahedron $(\mathrm{NBO} / \mathrm{T})$ of 2 in both cases. Note that the $\mathrm{NBO} / \mathrm{T}$ is related to the network connectivity used in other studies $\left(\mathrm{NC}=(4-(\mathrm{NBO} / \mathrm{T})) .{ }^{29} \mathrm{Si}\right.$ spectra of the 52S4.6 composition showed a much broader peak, which we have deconvoluted (Table 2). The two peaks were at 81 and $88 \mathrm{ppm}$, corresponding to chemical shifts for $\mathrm{Q}^{2}$ and $\mathrm{Q}^{3}$ species respectively. The area ratios of $\mathrm{Q}^{2}$ and $\mathrm{Q}^{3}$ species give the number of non-bridging oxygens/ silica tetrahedron, showing that there was no significant difference between the network connectivity of the glass plate and the fibers obtained from it.

Further results can be considered from the comparison among the NBO ratio per tetrahedra obtained from the NMR analysis with the theoretical structure calculated from: ${ }^{[26]}$

$$
\mathrm{NBO} / \mathrm{T}=2\left(\mathrm{Na}_{2} \mathrm{O}+\mathrm{CaO}-(3 m+2 n) \mathrm{P}_{2} \mathrm{O}_{5}\right) / \mathrm{SiO}_{2}
$$


where $m$ and $n$ are the molar proportions of ortho- and pyrophosphate species, whereas $\mathrm{Na}_{2} \mathrm{O}, \mathrm{CaO}, \mathrm{SiO}_{2}$ and $\mathrm{P}_{2} \mathrm{O}_{5}$ represent the molar percentages of each component. We calculated the $\mathrm{NBO} / \mathrm{T}$ ratios using the compositions acquired by $\mathrm{XRF}$ and the proportions of phosphate species calculated from deconvolution of ${ }^{31} \mathrm{P}$ NMR spectra, and the results are presented in Table 2 . There is relatively good agreement between the calculated NBO/T values from the chemical composition and those obtained from the

${ }^{29} \mathrm{Si}$ NMR spectra (Table 2). These results must be interpreted taking into account that the XRF analyses cannot be treated as a strictly quantitative analysis; therefore the data must be interpreted comparatively between bulk and fiber samples. In this way, we can verify that the slight differences in compositions between bulk glass plates and fibers observed by XRF agree with the subtle differences of the silicate structure calculated from the NMR analyses. Consequently, we can affirm that there is no other effect on the glass structure of the laser spinning process other than that promoted by the subtle change in composition.

\subsection{Bioactivity tests}

Several samples were incubated in simulated body fluid (SBF) for periods of 3, 6, 12, 24, 48, 120, and 240 hours and subsequently analyzed by FESEM and TEM. After 12 hours of immersion in SBF the fibers appear completely covered by a layer of foamy material, which was identified as amorphous calcium phosphate by energy dispersive X-ray spectroscopy (EDS) and SAED analyses carried out in the TEM. After 1 or 2 days of incubation, many fibers became thicker and translucent as can be observed in the TEM micrograph (Fig. 3) that was obtained for the fibers of composition 52S4.6 after immersion for 2 days. The nanofibers appeared to be covered by a wavy and porous -non dense- layer of calcium phosphate which increased their diameter. However, the core of these fibers was still discernible since a clear line separates the 
outer layer from the core of the primary fiber, this indicates that the fibers were hollow. FESEM analyses confirmed these observations: Figure 4.a presents a micrograph showing the appearance of a thin hollow fiber from the sample with composition 45S5 after 2 days of immersion. The core of the fiber has disappeared completely and, instead, a porous tube was observed. Some other fibers still keep their core, a solid fiber from the same sample is shown in Fig. 4.b, although had a similar porous structure along its surface. A similar porous structure appears on the surface of the fibers after $12 \mathrm{~h}$ of incubation (Fig. 4.c). Figure 4.d shows the detail of the porous structure formed on the surface of the fibers after 5 days of immersion, presenting the usual nodule morphology observed after hydroxyapatite precipitation. Elemental microanalyses by EDS in FESEM and TEM showed that both the hollow and the solid fibers were severely depleted in silicon. After $24 \mathrm{~h}$ of immersion in SBF the level of silicon detected is clearly below that of phosphorous and calcium and still undergoes further decay after $48 \mathrm{~h}$. The fibers were mainly composed of calcium phosphate with the amount of silicon reduced to a trace. Specifically, the $\mathrm{Si} / \mathrm{Ca}$ ratios among many analyses of different fibers after 24 hours of immersion fell well below unity, with great variation due to the limitations of the analysis technique and the diverse size of the fibers. Additionally, for immersion times of 48 hours and longer the $\mathrm{Si} / \mathrm{Ca}$ ratio is well below 0.1 -with no perceptible changes at longer times, which is negligible compared the $\mathrm{Si} / \mathrm{Ca}$ ratio in $45 \mathrm{~S} 5$ glass $(1.71)$. The patterns obtained by SAED show that the fibers and their calcium phosphate coatings were still mostly amorphous with faint evidences of a scarce presence of some nanocrystals in some fibers after 48 hours of incubation for both compositions.

The ion concentration change in the solution was evaluated using inductively coupled plasma (ICP) after incubation of the fibers in the SBF medium and the results are shown 
in Figure 5. The low levels of variation measured are due to the low quantity of glass fibers immersed in the solution so that the ratio of the surface area of the sample to volume of solution corresponded to the standards. The concentration of $\mathrm{Si}$ ions increased rapidly during the first 48 hours until it reached a maximum of approximately $4.0 \mu \mathrm{g} \mathrm{ml}^{-1}$ for the fibers with composition $45 \mathrm{~S} 5$, and $4.2 \mu \mathrm{g} \mathrm{ml}^{-1}$ for $52 \mathrm{~S} 4.6$ fibers. These values are very close to the ion concentration calculated to correspond to total dissolution of the fibers, which would be $4.2 \mu \mathrm{g} \mathrm{ml}^{-1}$ for $45 \mathrm{~S} 5$ glass and $4.74 \mu \mathrm{g} \mathrm{ml}^{-1}$ for the 52S4.6 composition. These results, together with the levels of silicon detected in the fibers, imply that total dissolution of the silica network occurred throughout the experiments. Conversely, the concentrations of $\mathrm{Ca}$ and $\mathrm{P}$ ions increased at the beginning of incubation, which agrees with a progressive dissolution of the fibers. Calcium ions are at non-bridging oxygen sites in the glass and undergo ion exchange with $\mathrm{H}^{+}$ions from the solution as the first stage of dissolution. ${ }^{[2]}$ Phosphate is present in the glass mainly as orthophosphate, charge balanced by calcium, which means it is not a part of the glass network and can also be lost to solution rapidly. ${ }^{[24]}$ Particularly, the concentration of $\mathrm{P}$ ions stays almost uniform between $12 \mathrm{~h}$ and $48 \mathrm{~h}$ for the $45 \mathrm{~S} 5$ fibers and between $12 \mathrm{~h}$ and $120 \mathrm{~h}$ for the 52S4.6 samples and then starts to decrease. Whereas the concentration of $\mathrm{Ca}$ ions presents a higher oscillation and begins to decrease after 48 hours of immersion of the 45S5 sample and 120 hours for the 52S4.6 glass. The reduction of the $\mathrm{Ca}$ and $\mathrm{P}$ concentrations are due to calcium phosphate layers forming on the glass. The earlier reduction in $\mathrm{P}$ concentration for the 45S5 indicated that the calcium phosphate layer forms more rapidly on its surface compared to 52s 4.6 , which correlates to the difference in their $\mathrm{NBO} / \mathrm{T}$ values. The dissolution of the fibers continued after the beginning of the formation of the layer of calcium phosphate on their surface, which explains the formation of the hollow fibers, as previously observed for 
45S5 microspheres. ${ }^{[27]}$ The dissolution rate of these fibers was very similar to that previously reported by H.-W. Kim et al. for glass nanofibers obtained from electrospinning of a sol-gel. ${ }^{[21]}$ This outcome is in clear agreement with previous results that relate the increase of dissolution rate of bioactive glasses with the decrease of their particle size. $^{[28]}$

Further analyses by TEM show that for an incubation time of 5 days, the layer of calcium phosphate presents a faint crystalline structure, since weak rings appeared in the SAED pattern for both compositions. After 10 days of incubation the crystalline structure is more pronounced. Detailed analysis of the porous layer on the surface of the fibers reveals the formation of needle-like nanocrystals (Fig. 6.a), with morphology very similar to that previously reported for apatite. ${ }^{[29,30]}$ The comparison of the brightfield and dark-field TEM micrographs presented in Figure 6 confirm their crystallinity, since light scattered by the thin crystals make them to appear brighter in the dark-field micrograph. Additionally, the SAED pattern presented in Figure 7 shows well defined rings which correspond to the presence of dispersed nanocrystals. These rings have been identified according to their good correlation with the interplanar distances of the hydroxyapatite. The first ring presents a preferred orientation which coincides with the growth of the crystals from the fiber surface, whereas the second ring appears thicker due to the addition of signals from three planes with very close spacing. The delay on the formation of the apatite layer in comparison with the usual rates for these bioactive glasses may be attributed to the short radius of curvature. ${ }^{[31]}$ To summarize, after incubation times in the order of 5 days the amorphous and porous layer of calcium phosphate covering the nanofibers crystallizes constituting a porous surface formed of nanocrystals of hydroxyapatite. 


\section{Conclusions}

Bioactive glass nanofibers with different melt-derived compositions were produced using a new technique which allows easy tailoring of their composition. The nanofibers were fully dense, free standing and amorphous with good consistency and flexibility which facilitates their handling, thus they can be post-processed for easy weaving or forming to adapt to complicated spaces.

After incubation in simulated body fluid their silica network was almost completely dissolved. The samples tested herein showed slight but perceptible differences in their dissolution rates according to the short variation of composition in their glass formulation. Between 5 to 10 days of incubation they changed into hollow nanostructures with a porous surface formed of nanocrystals of hydroxyapatite.

Consequently, Laser Spinning has been demonstrated as an efficient technique for the production of nanofibers of bioactive glasses and new nanostructures with potential for tissue engineering scaffolds; fillers in bone defects; reinforcing agents in nanocomposites. Adtionally, the mats produced are composed of separated nanofibers without strong adhesion forces among them, consequently this will allow their further processing into ordered arrays. Finally, the capability of the laser spinning technique to produce nanofibers with a wide range of compositions makes evident its potential to produce nanofibers with different rates of bioresorption to control the release of active ions that have the potential to stimulate the gene expression and cellular response necessary for tissue regeneration. 


\section{Experimental}

Laser Spinning of nanofibers: The precursor materials employed in this work for the Laser Spinning process were a series of glass plates with the compositions of Bioglass ${ }^{\circledR}$ 45s5 and 52s4.6. Several pieces of each glass composition were produced by means of the traditional method of glass melting. A blend of the raw materials (soda, lime, silica and phosphorous oxide) was melted in a platinum crucible at $1500{ }^{\circ} \mathrm{C}$ using an electric furnace. The molten glass was poured on a steel mould to form plates having a thickness of $6 \mathrm{~mm}$, and rapidly introduced into another furnace, prewarmed to $500^{\circ} \mathrm{C}$, for annealing before furnace cooling to ambient temperature. The glass plates were laser processed using a high power $\mathrm{CO}_{2}$ laser (Rofin Sinar DC 035) emitting, in continuous mode, a beam of infrared radiation with wavelength of $10.6 \mu \mathrm{m}$ and power of $2.5 \mathrm{~kW}$. The laser beam was focused using a lens with a focal length of $127 \mathrm{~mm}$ at a fixed distance of $8 \mathrm{~mm}$ above the surface of the workpiece. The solid plate was transversally displaced at a speed of $4 \mathrm{~mm} \mathrm{~s}^{-1}$ by means of a motor driven translation stage controlled by a computer. At the same time, an off-axis supersonic nozzle operating at a nominal pressure of $1.210^{6} \mathrm{~Pa}$ generated the high speed gas jet whereby the molten material was removed. A coaxial convergent nozzle at $410^{5} \mathrm{~Pa}$ produced a protective flow to avoid damage of the lens. Compressed air was used for the supersonic jet and argon for the coaxial protective flow. A cotton-like mesh of free-standing fibers is produced by this method.

Characterization of the fibers: The characterization of the morphology was carried out using a field emission scanning electron microscopy (JEOL JSM-6700f) and transmission electron microscopy (JEOL JEM-2010 FEG). The compositions of the 
precursor glass plates and the fibers obtained from them were analyzed by XRF under the same conditions of analysis and sample preparation to avoid any distortion from differences in sample quantity, size or morphology. In this sense, $0.1 \mathrm{~g}$ of the glass plate and glass fibers from every sample was ground to the same size. The powdered samples were mixed with a flux of lithium borate, heated in a crucible and cast in a mould to produce a homogeneous glass bead. Semi-quantitative analyses were performed, since no proper standard for calibrating the XRF measurements were found. However, this method yields a reliable comparison of the relative proportions in the glasses and their corresponding fibers, since sample preparation and XRF analyses were performed in identical conditions. The glass structure was analyzed by means of magic angle spinning NMR. Solid-state ${ }^{29} \mathrm{Si}$ and ${ }^{31} \mathrm{P}$ spectra were recorded on a Bruker Avance DSX300 spectrometer at the resonance frequency of 59.63 and $121.51 \mathrm{MHz}$, respectively. The glass powder was packed in a Bruker $4 \mathrm{~mm}$ MAS probe and then spun at $7 \mathrm{kHz}$. Both spectra were obtained with a $8.7 \mu$ s pulse length and a recycle delay of $12 \mathrm{~s}$ between pulses. Chemical shift for ${ }^{29} \mathrm{Si}$ spectra were referenced to tetramethylsilane in parts per million (ppm) and to a $50 \%$ aqueous solution of $\mathrm{H}_{3} \mathrm{PO}_{4}$ for ${ }^{31} \mathrm{P}$.

Bioactivity tests: In vitro bioactivity of the fibers was tested in Simulated Body Fluid $(\mathrm{SBF})$. First we measured the surface area to mass ratio of the fibers with a gas adsorption analyzer to estimate the mass of the sample necessary to obtain a specific surface area. Then, the volume of SBF and mass of the sample were chosen to keep the ratio of surface area of the sample to volume of SBF equal to $10 \mathrm{~mm}^{2} \mathrm{ml}^{-1}$ [32]. Then the fibers (approximately $6 \mathrm{mg}$ ) were immersed in $300 \mathrm{ml}$ of SBF and incubated for periods of $3,6,12,24,48,120$, and 240 hours at $36.5^{\circ} \mathrm{C}$ in line with optimized conditions for testing bioactive glass in SBF [33]. The fibers were then removed from the solution, gently washed to terminate the reaction, and dried at ambient temperature 
in a desiccator. The samples were subsequently analyzed by FESEM and TEM and the ion concentration in the solution was evaluated by optical emission spectroscopy inductive coupled plasma spectroscopy (ICP-OES, Perkin Elmer).

Received: ((will be filled in by the editorial staff))

Revised: ((will be filled in by the editorial staff)) Published online: ((will be filled in by the editorial staff))

[1] R. Langer, J. P. Vacanti, Science 1993, 260, 920.

[2] L. L. Hench, J. M. Polak, Science 2002, 295.

[3] B. D. Ratner, S. J. Bryant, Annu. Rev. Biomed. Eng. 2004, 6, 41.

[4] S. J. Hollister, Nat. Mat. 2005, 4, 518.

[5] M. M. Stevens, Mater. Today 2008, 11, 18.

[6] B. M. Hatcher, C. A. Seegert, A. B. Brennan, J. Biomed. Mater. Res. 2003, 66A, 840.

[7] R. L. Orefice, L. L. Hench, A. E. Clark, A. B. Brennan, J. Biomed. Mater. Res. 2001, 55, 460 .

[8] T. Peltola, M. Jokinen, S. Veittola, H. Rahiala, A. Yli-Urpo, Biomaterials 2001, $22,589$.

[9] M. Marcolongo, P. Ducheyne, W. C. LaCourse, J. Biomed. Mater. Res. 1997, 37,440 .

[10] D. C. Clupper, J. E. Gough, M. M. Hall, A. G. Clare, W. C. LaCourse, L. L. Hench, J. Biomed. Mater. Res. 2003, 67A, 285.

[11] R. F. Brown, D. E. Day, T. E. Day, S. Jung, M. N. Rahaman, Q. Fu, Acta Biomater. 2008, 4, 387.

[12] I. Ahmed, M. Lewis, I. Olsen, J. C. Knowles, Biomaterials 2004, 25, 501.

[13] E. Pirhonen, H. Niiranen, T. Niemela, M. Brink, P. Tormala, J. Biomed. Mater. Res. Part B 2006, 77B, 227.

[14] D. C. Clupper, J. E. Gough, P. M. Embanga, I. Notingher, L. L. Hench, M. M. Hall, J. Mater. Sci.-Mater. Med. 2004, 15, 803.

[15] F. T. Wallenberger, in Advanced inorganic fibers: processes, structures, properties, applications., (Ed: F. T. Wallenberger), Kluwer Academic Publishers, Boston 2000, 123.

[16] F. Quintero, A. B. Mann, J. Pou, F. Lusquiños, A. Riveiro, Appl. Phys. Lett. 2007, 90 .

[17] F. Quintero, J. Pou, F. Lusquiños, A. Riveiro, Appl. Surf. Sci. 2007, 254, 1042.

[18] M. M. Stevens, J. H. George, Science 2005, 310, 1135.

[19] W. J. Li, C. T. Laurencin, E. J. Caterson, R. S. Tuan, F. K. Ko, J. Biomed. Mater. Res. 2002, 60, 613.

[20] T. J. Sill, H. A. von Recum, Biomaterials 2008, 29, 1989.

[21] H. W. Kim, H. E. Kim, J. C. Knowles, Adv. Funct. Mater. 2006, 16, 1529.

[22] W. Xia, D. M. Zhang, J. Chang, Nanotechnology 2007, 18, 7.

[23] A. Tilocca, A. N. Cormack, N. H. de Leeuw, Faraday Discuss. 2007, 136, 45.

[24] I. Elgayar, A. E. Aliev, A. R. Boccaccini, R. G. Hill, J. Non-Cryst. Solids 2005, $351,173$.

[25] M. W. G. Lockyer, D. Holland, R. Dupree, J. Non-Cryst. Solids 1995, 188, 207. 
[26] H. Grussaute, L. Montagne, G. Palavit, J. L. Bernard, J. Non-Cryst. Solids 2000, $263,312$.

[27] S. Radin, P. Ducheyne, S. Falaize, A. Hammond, J. Biomed. Mater. Res. 2000, 49, 264.

[28] P. Sepulveda, J. R. Jones, L. L. Hench, J. Biomed. Mater. Res. 2002, 61, 301.

[29] H. M. Kim, T. Himeno, T. Kokubo, T. Nakamura, Biomaterials 2005, 26, 4366.

[30] H. Takadama, H. M. Kim, T. Kokubo, T. Nakamura, J. Biomed. Mater. Res.

2001, 57, 441 .

[31] J. R. Jones, L. L. Hench, Mater. Sci. Technol. 2001, 17, 891.

[32] T. Kokubo, H. Takadama, Biomaterials 2006, 27, 2907.

[33] J. R. Jones, P. Sepulveda, L. L. Hench, J. Biomed. Mater. Res. 2001, 58, 720. 


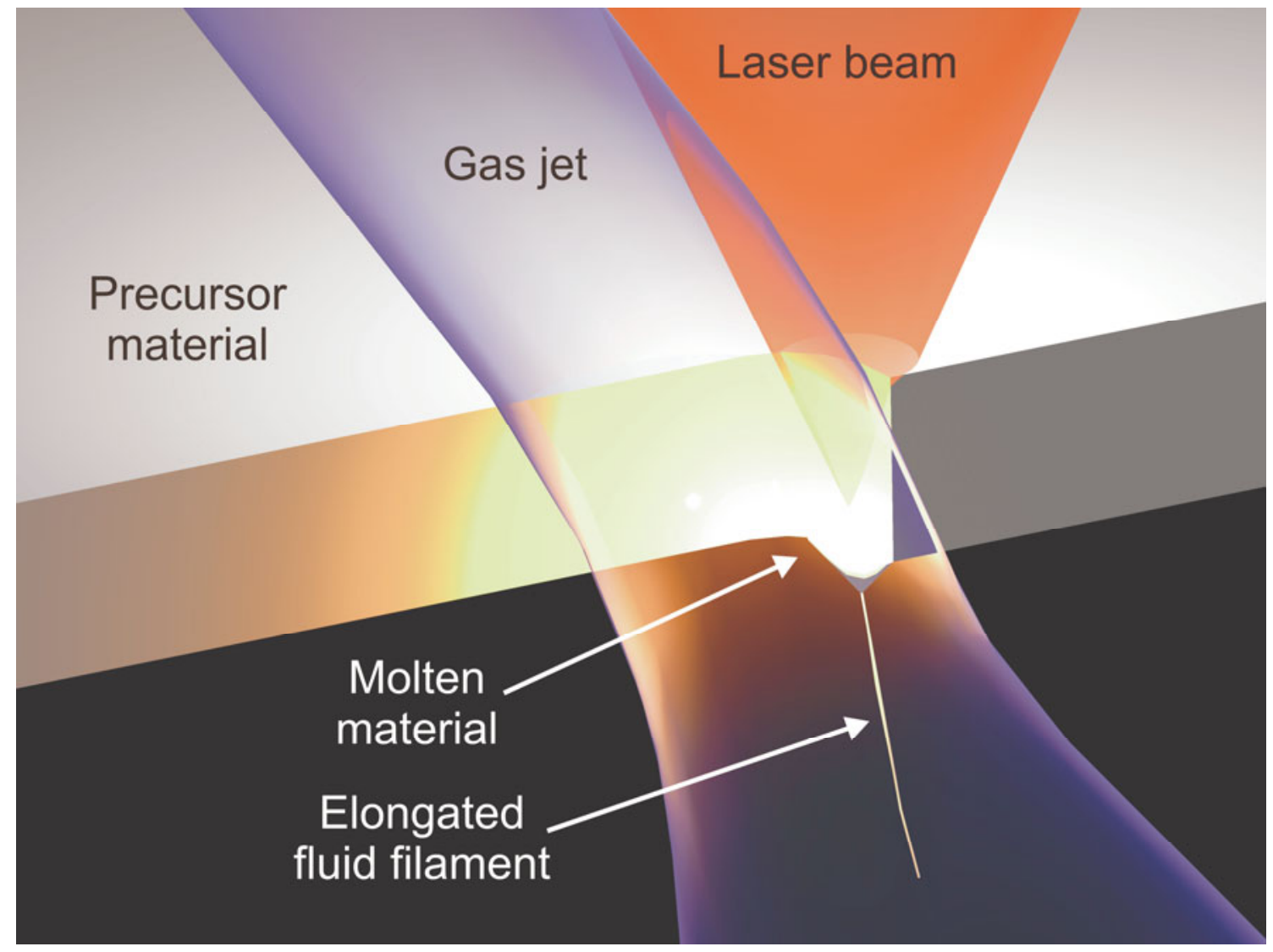

Figure 1. The Laser Spinning process involves the use of a high power laser to melt a very small volume of the precursor material. A high speed supersonic gas jet causes the rapid elongation and cooling of the melt. 


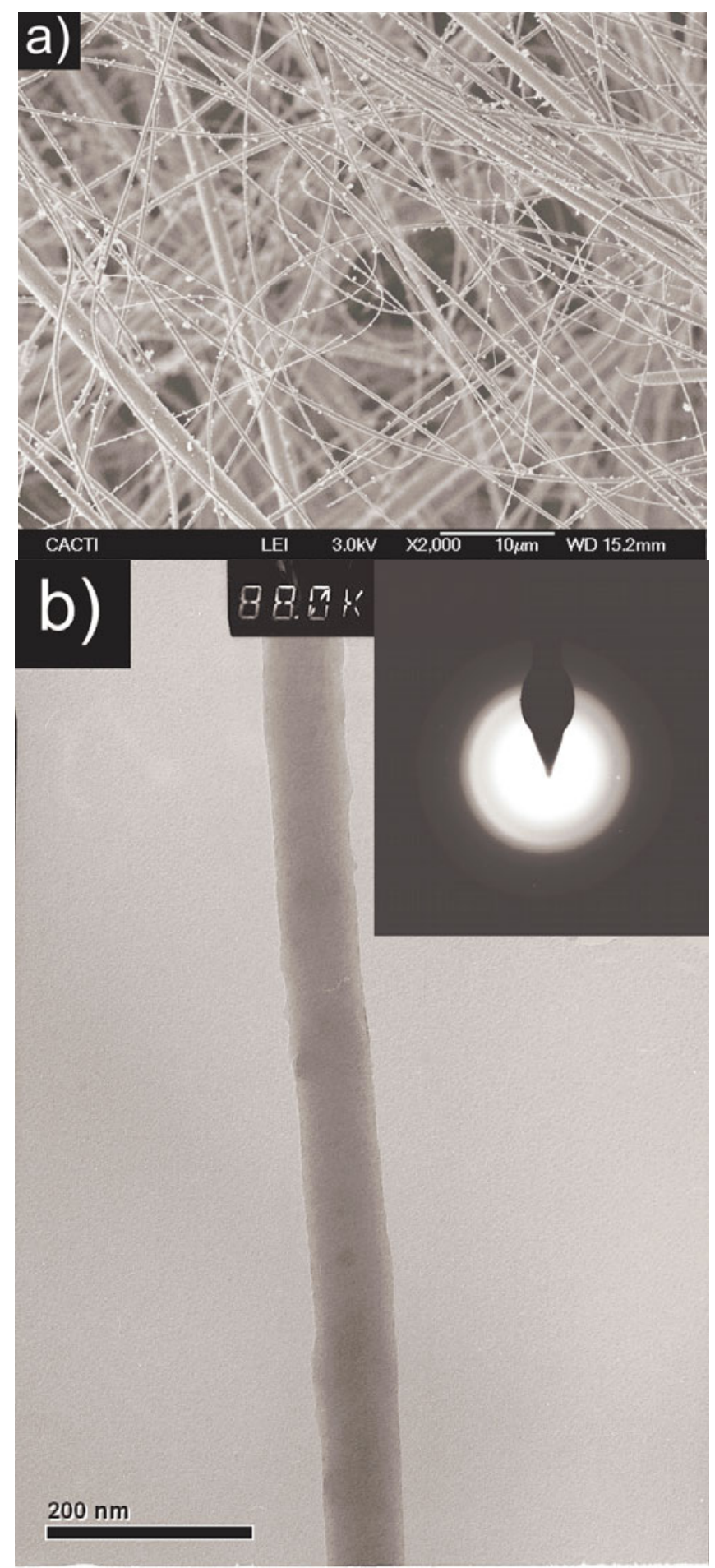

Figure 2. (a) FESEM micrograph showing the typical appearance of the as-produced fibers. (b) TEM micrograph of a nanofiber of bioactive glass with the composition 52S4.6, it appears with uniform cylindrical section, solid and smooth. The inset presents the SAED showing its amorphous structure. 


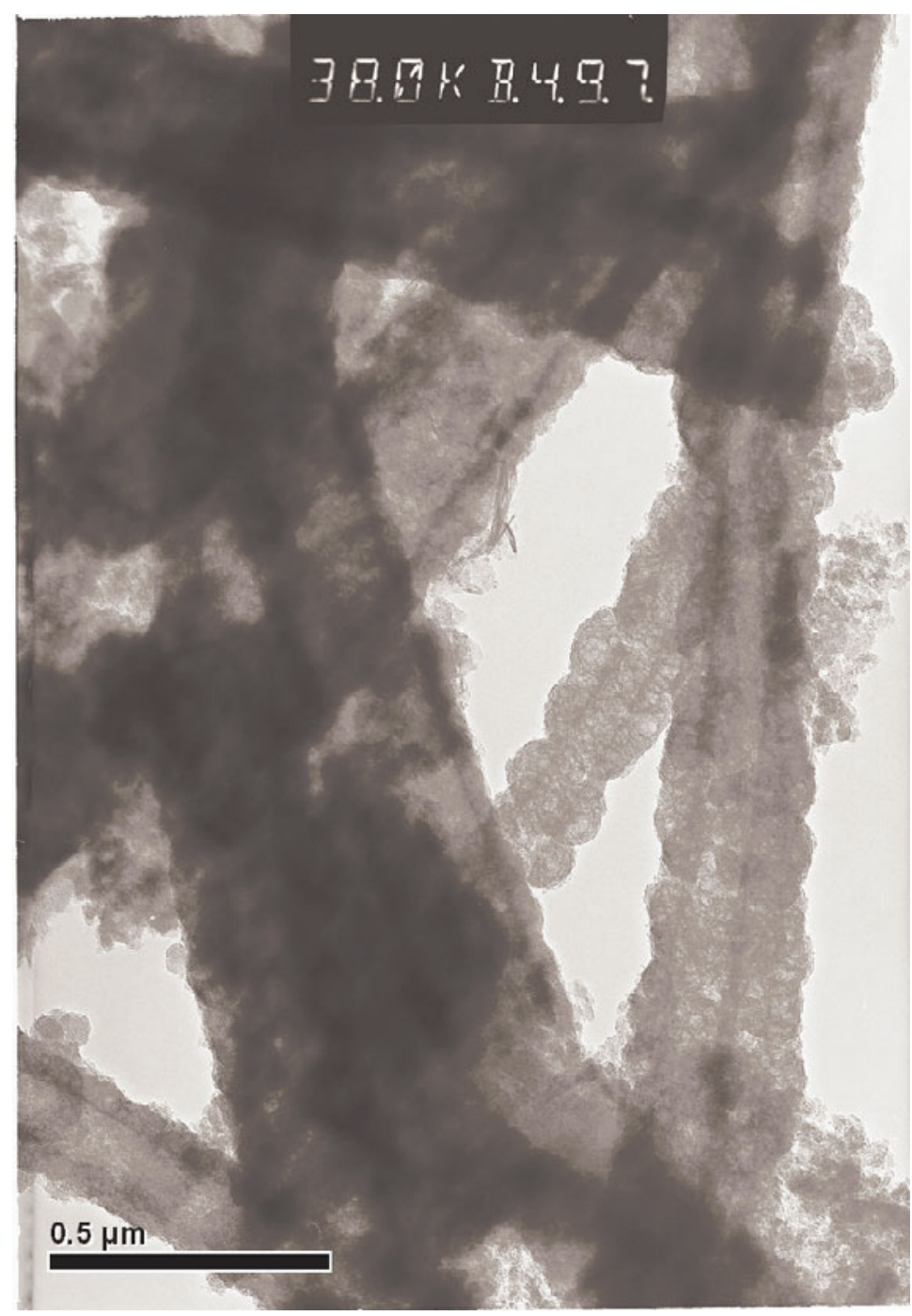

Figure 3. TEM micrograph of nanofibers with composition $52 \mathrm{~S} 4.6$ after immersion in SBF for 2 days. 


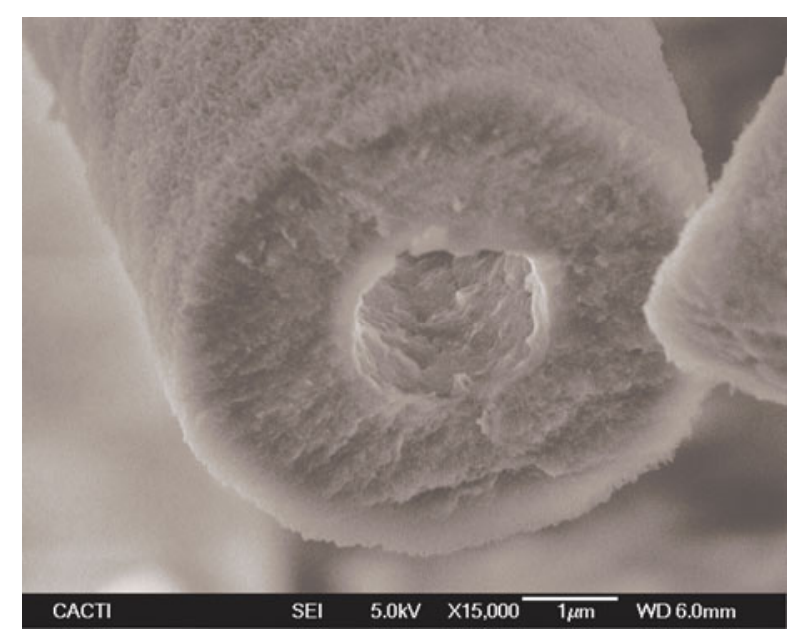

a)

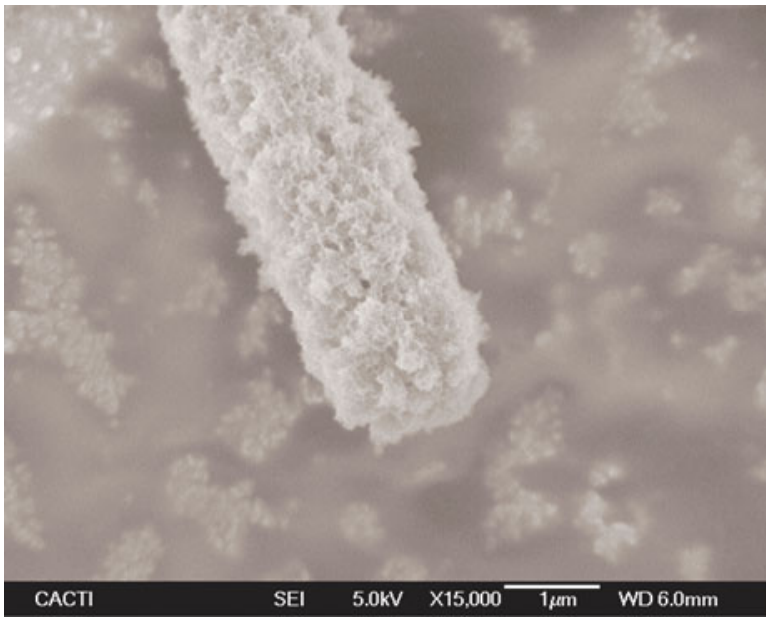

c)

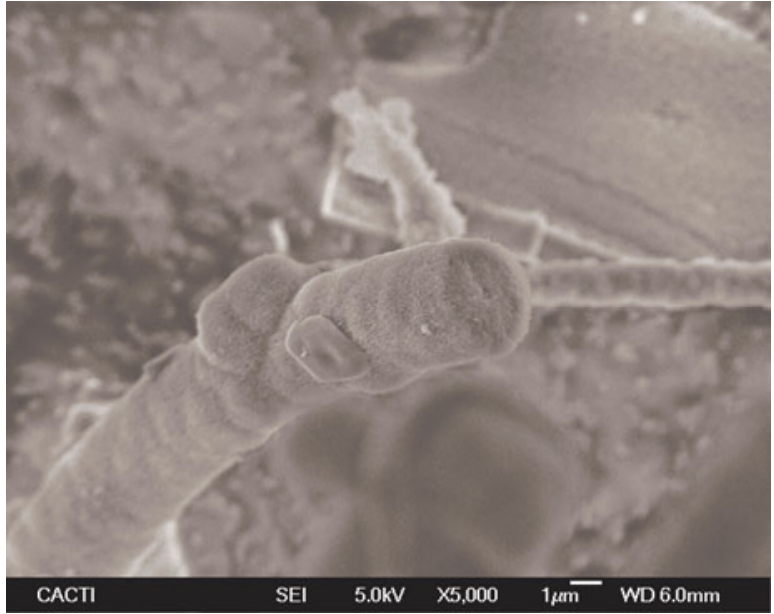

b)

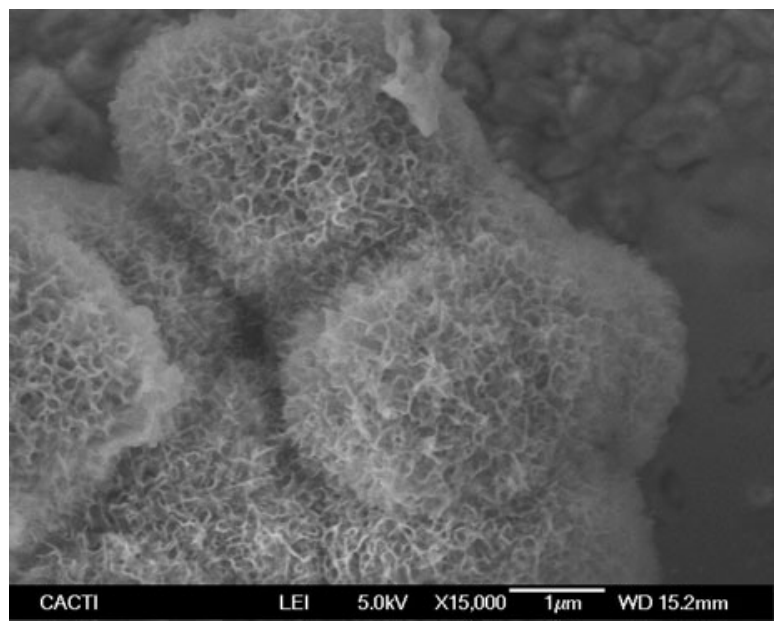

d)

Figure 4. FESEM micrographs of the fibers with composition 45S5 after: a) and b) 48 hours of immersion; c) 12 hours; and d) 5 days. 

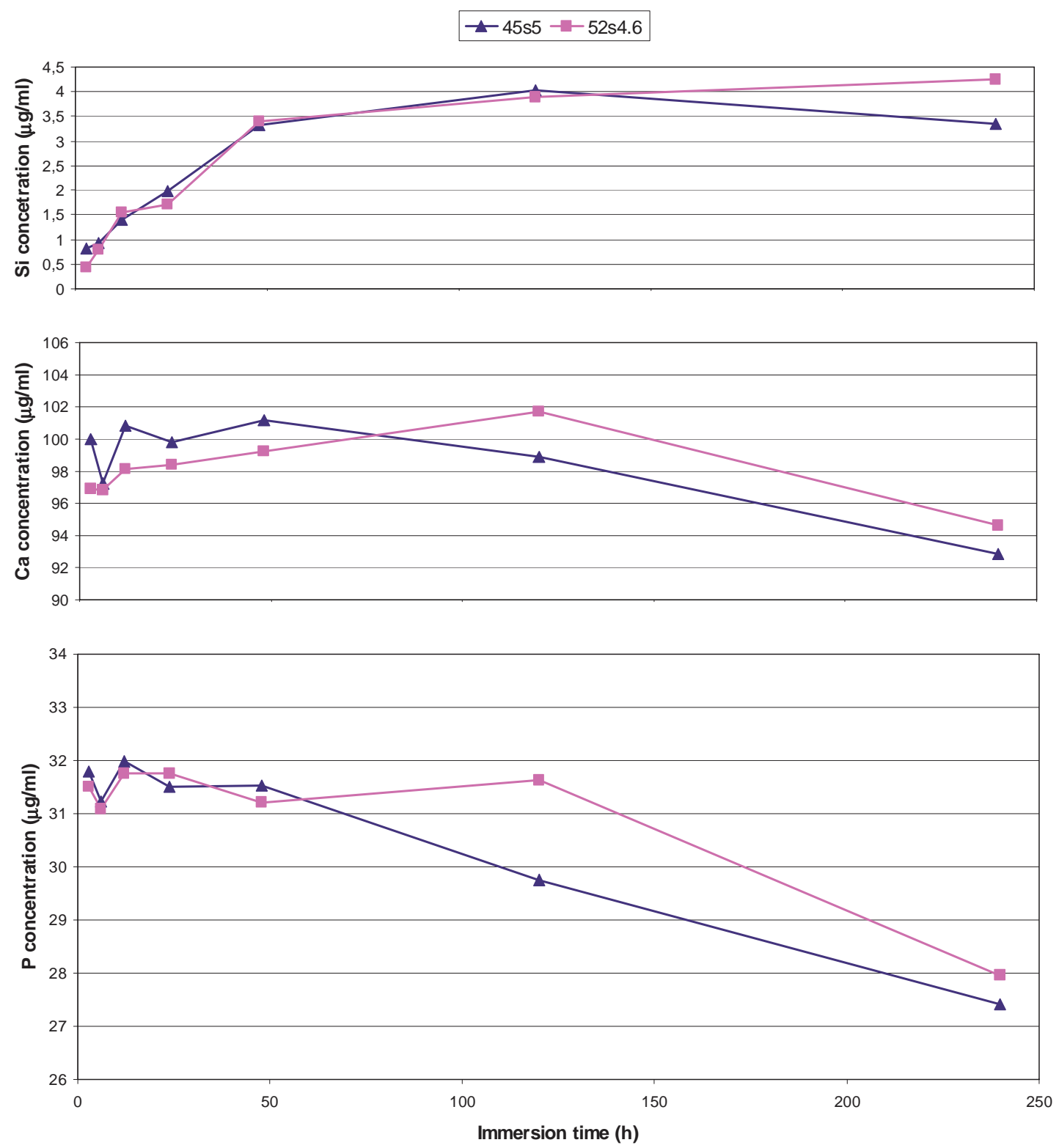

Figure 5. Elemental concentration measured by ICP analysis of solution medium after incubation in SBF of the fibers with compositions 45S5 and 52S4.6. 


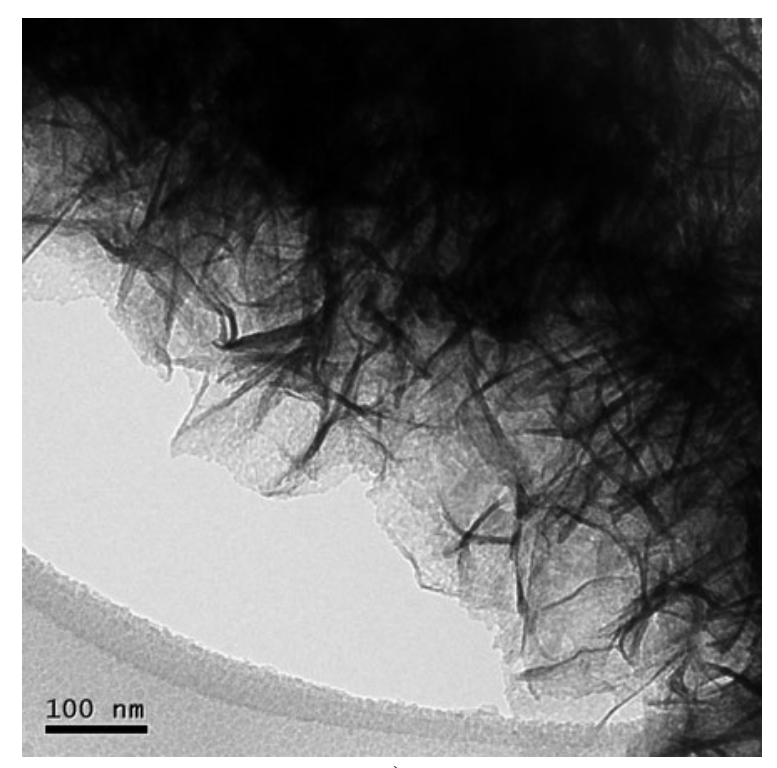

a)

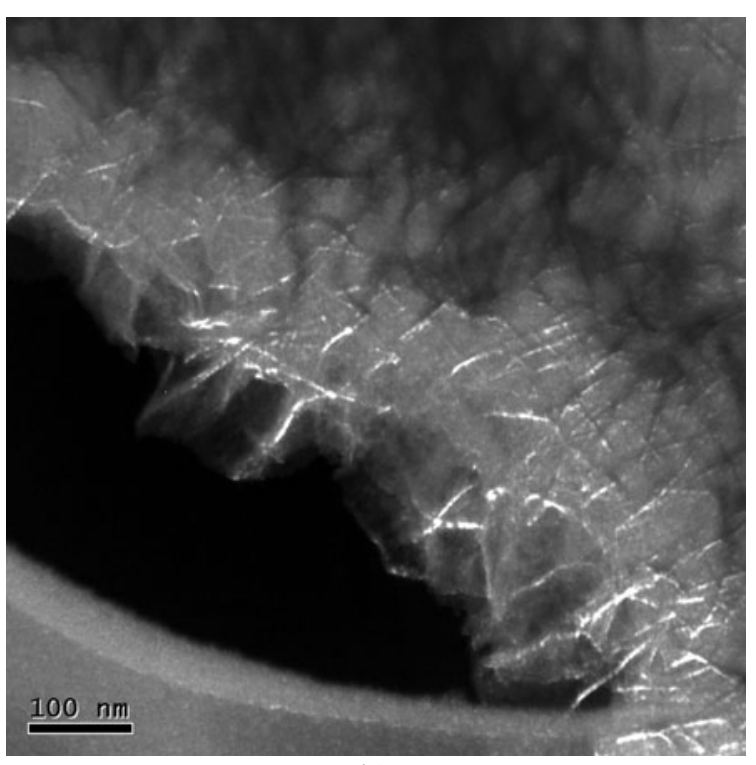

b)

Figure 6. TEM micrographs showing a detail of the porous surface of a fiber with composition 45S5 after 10 days of immersion in SBF. The bright-field a) and dark-field b) images confirm the presence of nanocrystals. 


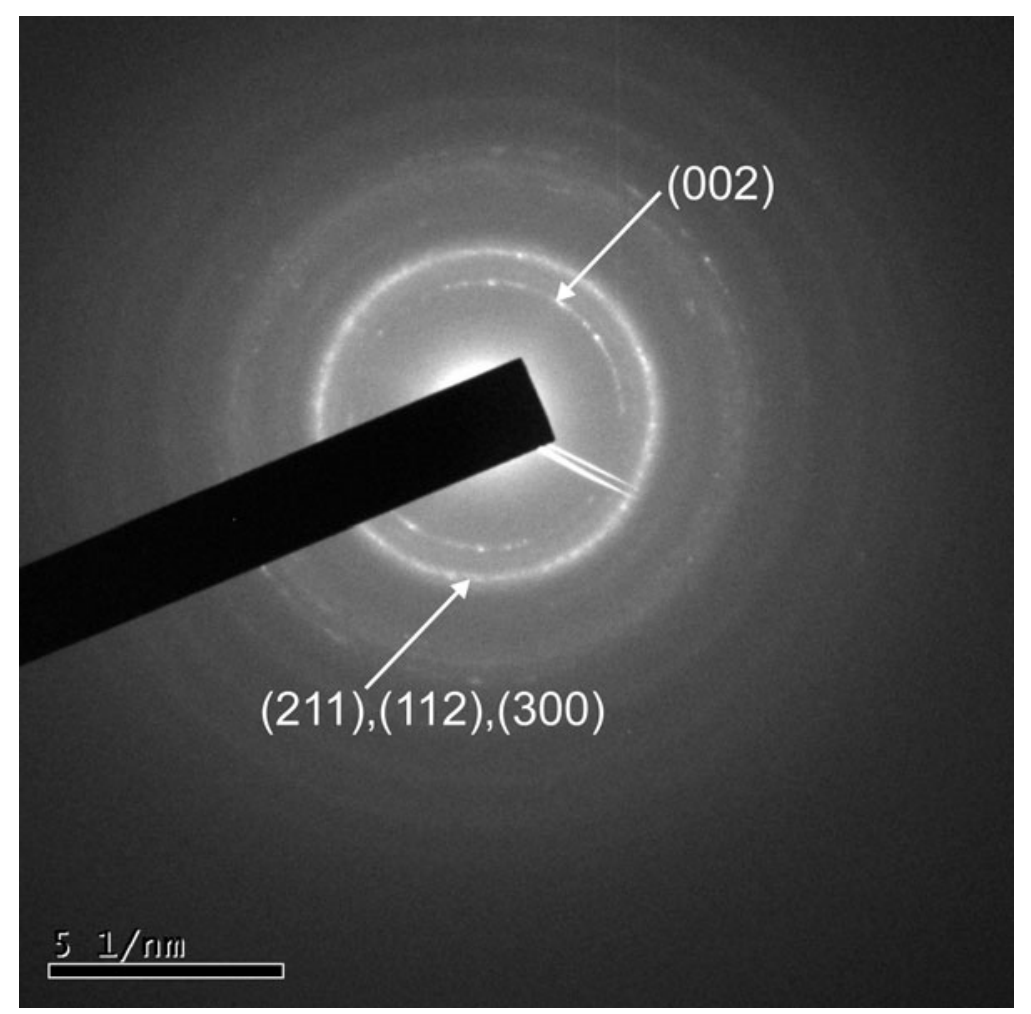

Figure 7. SAED pattern of the nanocrystals on the surface of a fiber with composition 45S5 after 10 days in SBF. The positions of the rings coincide with interplanar distances for hydroxyapatite. 
Table 1. Compositions in molar percentage of the glass plates and the fibers produced from them.

\begin{tabular}{|c|c|c|c|c|c|c|}
\hline & $\begin{array}{c}\text { 45s5- } \\
\text { nominal }\end{array}$ & $\begin{array}{c}\text { 45s5- } \\
\text { bulk }\end{array}$ & $\begin{array}{c}\text { 45s5- } \\
\text { fibers }\end{array}$ & $\begin{array}{c}\text { 52s4.6- } \\
\text { nominal }\end{array}$ & $\begin{array}{c}\text { 52s4.6- } \\
\text { bulk }\end{array}$ & $\begin{array}{c}\text { 52s4.6- } \\
\text { fibers }\end{array}$ \\
\hline $\mathrm{SiO}_{2}$ & 46.1 & 45.54 & 46.00 & 52.1 & 51.47 & 52.27 \\
\hline $\mathrm{Al}_{2} \mathbf{O}_{3}$ & - & 0.40 & 0.46 & - & 0.48 & 0.45 \\
\hline $\mathrm{CaO}$ & 26.9 & 27.42 & 27.68 & 23.8 & 24.13 & 24.71 \\
\hline $\mathrm{P}_{2} \mathrm{O}_{5}$ & 2.6 & 2.55 & 2.43 & 2.6 & 2.53 & 2.30 \\
\hline $\mathrm{Na}_{2} \mathrm{O}$ & 24.4 & 23.87 & 23.19 & 21.5 & 21.09 & 20.00 \\
\hline $\mathrm{K}_{2} \mathrm{O}$ & - & 0.18 & 0.19 & - & 0.26 & 0.21 \\
\hline $\mathrm{Fe}_{2} \mathrm{O}_{3}$ & - & 0.01 & 0.02 & - & 0.02 & 0.01 \\
\hline $\mathrm{TiO}_{2}$ & - & 0.03 & 0.03 & - & 0.03 & 0.03 \\
\hline
\end{tabular}

Table 2. Results from the deconvolution of the ${ }^{29} \mathrm{Si}$ spectra. The silicate structure obtained from them are presented showing the NBO/T values. The NBO/T values are also estimated from the compositional analyses carried out by XRF and results of orthoand pyrophosphate species obtained from the ${ }^{31} \mathrm{P}$ NMR spectra.

\begin{tabular}{|c|c|c|c|c|c|}
\hline Sample & $\begin{array}{c}\text { Peak position } \\
{[\mathrm{ppm}]}\end{array}$ & $\begin{array}{c}\text { FWHM } \\
{[\mathrm{ppm}]}\end{array}$ & Area ratio & $\mathrm{NBO} / \mathrm{T}[\mathrm{a}]$ & $\mathrm{NBO} / \mathrm{T}[\mathrm{b}]$ \\
\hline 45S5 bulk & -79.5 & 6.3 & 100 & 2 & 1.93 \\
\hline 45S5 fibers & -79.5 & 6.3 & 100 & 2 & 1.91 \\
\hline \multirow{2}{*}{$52 \mathrm{~S} 4.6$ bulk } & -80.7 & 9.7 & 44.3 & \multirow{2}{*}{1.44} & \multirow{2}{*}{1.48} \\
\hline & -88.2 & 12.1 & 55.7 & & \\
\hline \multirow{2}{*}{$52 \mathrm{~S} 4.6$ fibers } & -80.7 & 10.6 & 41.5 & \multirow{2}{*}{1.41} & \multirow{2}{*}{1.47} \\
\hline & -88.3 & 13.3 & 58.5 & & \\
\hline
\end{tabular}

[a] NBO/T value obtained from the ${ }^{29} \mathrm{Si}$ spectra. [b] NBO/T value obtained from the XRF analyses. 
The production of nanofibers of bioactive glass by laser spinning is reported. The technique involves melting and elongation of a precursor material with tailored composition using a high power laser and a supersonic gas jet. The morphology, composition and structure of the nanofibers are analyzed and their bioactivity is assessed by immersion in SBF.

Keywords: Tissue Engineering, bioactive glass, fibers, laser processing.

By Félix Quintero*, Juan Pou, Rafael Comesaña, Fernando Lusquiños, Antonio Riveiro, Adrian B. Mann, Robert G. Hill, Zoe Y. Wu, and Julian R. Jones

Laser Spinning of bioactive glass nanofibers

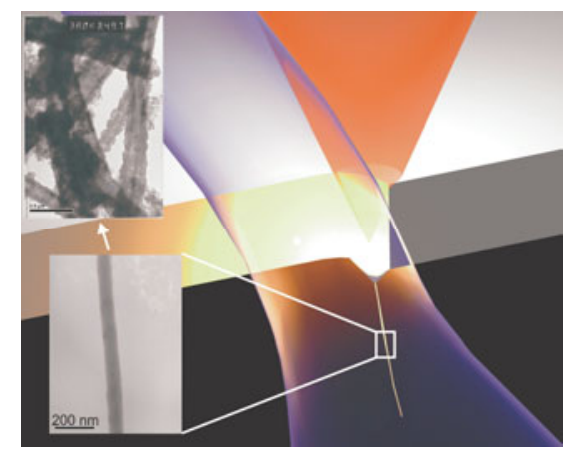

Column Title: F. Quintero et al./Laser Spinning Bioglass nanofibers 